\title{
Trend analysis of the wave storminess: the wave direction
}

\author{
M. Casas-Prat ${ }^{1,2}$ and J. P. Sierra ${ }^{1,2}$ \\ ${ }^{1}$ Laboratori d'Enginyeria Marítima, UPC, Barcelona, Spain \\ ${ }^{2}$ Centre Internacional d'Investigació dels Recursos Costaners, Barcelona, Spain
}

Received: 12 February 2010 - Revised: 14 June 2010 - Accepted: 15 July 2010 - Published: 25 August 2010

\begin{abstract}
In this paper changes in the wave pattern of the Catalan coast are analyzed in terms of wave storminess and wave direction based on series of 44 years of hindcast wave data. The analysis is performed employing two different techniques: one resulting from the combination of regression and bootstrapping and the other applying a Bayesian method. Although the trends are, in general, null, in some locations there are clear changes in the wave directional frequencies. These changes can significatively affect coastal hydrodynamics and morphodynamics.
\end{abstract}

\section{Introduction}

As a result of the greenhouse effect, increases in the mean global air and ocean temperatures are expected. Owing to more complex interactions of the atmosphere processes, changes in the wave storminess patterns are expected too. The final consequences in the coastal engineering field may be, therefore, noticeable variations in the coastal hydrodynamics and morphological typical conditions.

The uncertainty concerning the prediction of wave heights is large. In particular, stormy conditions are especially important because they are closely related to more hazardous events. Therefore, it becomes necessary to identify and quantify possible existing tendencies of storm wave related parameters.

Often only the maximum wave height and storm duration are analysed, remaining the wave direction in a second term. Note that a possible rotation of the mean wave direction may involve severe complications since most beach and harbour defence structures have been designed assuming the present wave incidences. A possible consequence would be a decrease of the harbour operability: increased frequency of coastal storms in the same direction that the harbour mouth orientation would influence the port operations.

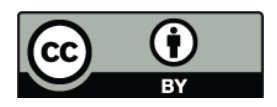

Correspondence to: M. Casas-Prat (merce.casas@upc.edu)
The present study, which focuses in the Catalan coast (NW Mediterranean Sea), aims to improve the present knowledge of the wave storminess variations at regional scale, specially focusing on the wave directionality.

\section{Material and methods}

\subsection{Data}

Due to the scarcity of long measured time series, this study is based on simulations: 44 years of hindcast wave data (19582001) from the European HIPOCAS project (Guedes Soares et al., 2002). Despite the limitations derived from the modelling compared to real observations, this is a widely used database (Gomis et al., 2008; Mösso et al., 2009, etc.) which has been validated (e.g. Musić and Nicković, 2008; Ratsimandresy et al., 2008). In spite of underestimating some extreme events, this dataset appears to be useful to study the long-term behaviour. It includes the entire Mediterranean basin and consists of 3 hourly simulated data, including typical wave parameters, from which the significant wave height $\left(H_{s}\right)$ and the mean wave direction $(\theta)$ are taken. For this study 40 nodes have been selected along the Catalan coast (resolution of $12.5 \mathrm{~km}$ approx.).

\subsection{Methodology}

The used methodology comprises mainly two aspects: i) the initial data management, and ii) the posterior trend analysis.

For storm definition, using the common Peak Over Threshold (POT) method, a primary $H_{S}$ threshold of $2 \mathrm{~m}$ is selected with a minimum duration requirement of $6 \mathrm{~h}$. In addition, with the aim of only considering statistically independent storms, the assumptions of Mendoza and Jiménez (2006) are followed.

In order to combine both $H_{s}$ and storm duration $(D)$, the storm energy content $(E)$ is used (Mendóza and Jiménez, 2006) as the representative parameter of the wave storminess. As the main interest of this study is the long term and not

Published by Copernicus Publications on behalf of the European Geosciences Union. 
seasonal variability, only its annual values (mean and maximum) are considered.

To examine $\theta$ long term variability, the $\theta$ associated to the peak of the storm is considered and it is classified according to the eight following sectors: N, NE, E, SE, S, SW, W, $\mathrm{NW}$. On the one hand, the evolution of the energy per each different sector is estimated and, on the other hand, the evolution of each sector frequency itself is studied by using the probability of occurrence approximated by Bayesian inference (Agresti, 2002).

Another important consideration before performing the trend analysis concerns the data scale. For not real data, a previous transformation is advisable in order not to measure distorted distances. This is the case of i) positive data (energy) and ii) compositional data (directional frequency) which is defined in the simplex space: a link between frequencies exists since they have constant sum and, therefore, statistical analysis (e.g. covariance matrix) based on the original percentages would contain spurious effects because of the inherent relation among them. To overcome this problem, for i) only a logarithmic transformation is applied whereas for ii) a more sophisticated expression is used, the isometric logratio (ilr) transformation (Egozcue et al., 2003). The last transformation translates the 8 directional percentages $\left\{p_{1}, \ldots, p_{8}\right\}$ into 7 coordinates $\left\{y_{1}, \ldots, y_{7}\right\}$ on an orthonormal basis in a real vector space $\mathfrak{R}^{7}$, obtained from sequential binary partition $\left(R_{i}, S_{i}\right)$. The conversion can be expressed as the log-ratio between the geometric mean of each partition $\left(g\left(p_{\in R_{i}}\right), g\left(p_{\in S_{i}}\right)\right)$ multiplied by a normalising factor $a\left(r_{i}, s_{i}\right)$ :

$$
y_{i}=a\left(r_{i}, s_{i}\right) \ln \left(\frac{g\left(p_{\in R_{i}}\right)}{g\left(p_{\in}\right)}\right), \quad a\left(r_{i}, s_{i}\right)=\sqrt{\frac{r_{i} s_{i}}{r_{i}+s_{i}}}, \text { for } i=
$$
$1, \ldots, 7$ where $r_{i}$ and $s_{i}$ are the number of elements in each partition.

The trend analysis methodology has been carefully chosen in order to be sufficiently accurate. It is a two-step method: i) trend detection and ii) trend quantification, being the directional frequency treated slightly different due to its additional complexity. For i) the Mann Kendall test is performed to detect the nodes with significant tendency. The chosen value for the significant level is 0.1 , slightly higher than the typical value of 0.05 in order not to miss any important trend.

For trend quantification (ii, applied to such interesting nodes with previously detected trend), a classical linear regression analysis is carried out and it is complemented with the bootstrap technique in order to assign measures of accuracy of statistical estimates. For this study a reasonable number of 1000 simulated samples are computed from which the marginal confidence intervals are directly obtained as the observed percentiles. Note that the regression analysis is carried out with the scale-transformed variables and, afterwards, the inverse conversion is done to properly interpret the results.

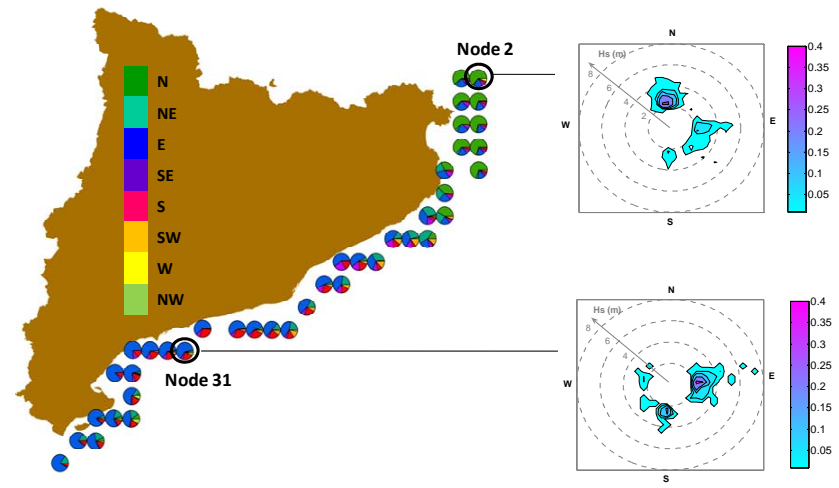

Fig. 1. Left: circular graphs of wave direction frequency along the Catalan coast in the period 1958-2001 (areas proportional to the percentages). Right: two examples of joint probability density function of each couple of Hs and wave direction.

A complementary analysis is performed by comparing the above trend quantification method (linear regression + bootstrap) with Bayesian analysis in order to better assess the uncertainty. This is limited to the energy analysis, because the difference between the two methods is found not to be significant (see Section 3.2). As it is well-known, the Bayesian inference assumes that the parameters to be estimated are random variables (in contrast to the frequentist alternative) According to the Bayes theorem, the (posterior) probability density function (pdf) of such unknown parameters is proportional to the product of the likelihood function and the prior pdf of such parameters. The likelihood involves the information given by the sample and the prior distribution has to be selected according to the physical problem characteristics. In this case, the data are assumed to be Gaussian distributed (reasonable since the energy is previously log-transformed) with a linear increasing mean. For the priors, flat uniform distributions are chosen.

To compute a sample of the posterior distributions, the Gibbs sampling method is used. Finally, the above explained Bayesian model is validated by sampling 1,000 times the original data using the obtained posterior distribution, in which the trend and the maximum values are compared between original and simulated data.

\section{Results and discussion}

\subsection{Wave direction characterisation}

As explained in the previous section, a first review of the predominant wave directions in the Catalan coast has been carried out in order to better evaluate the relative importance of possible existing long term tendencies.

Firstly, in terms of frequency (see Fig. 1), there is a general predominance of $\mathrm{E}$ direction ( $\mathrm{S}$ in a second term) except in the northern Catalan coast, in which $\mathrm{N}$ storms are more 


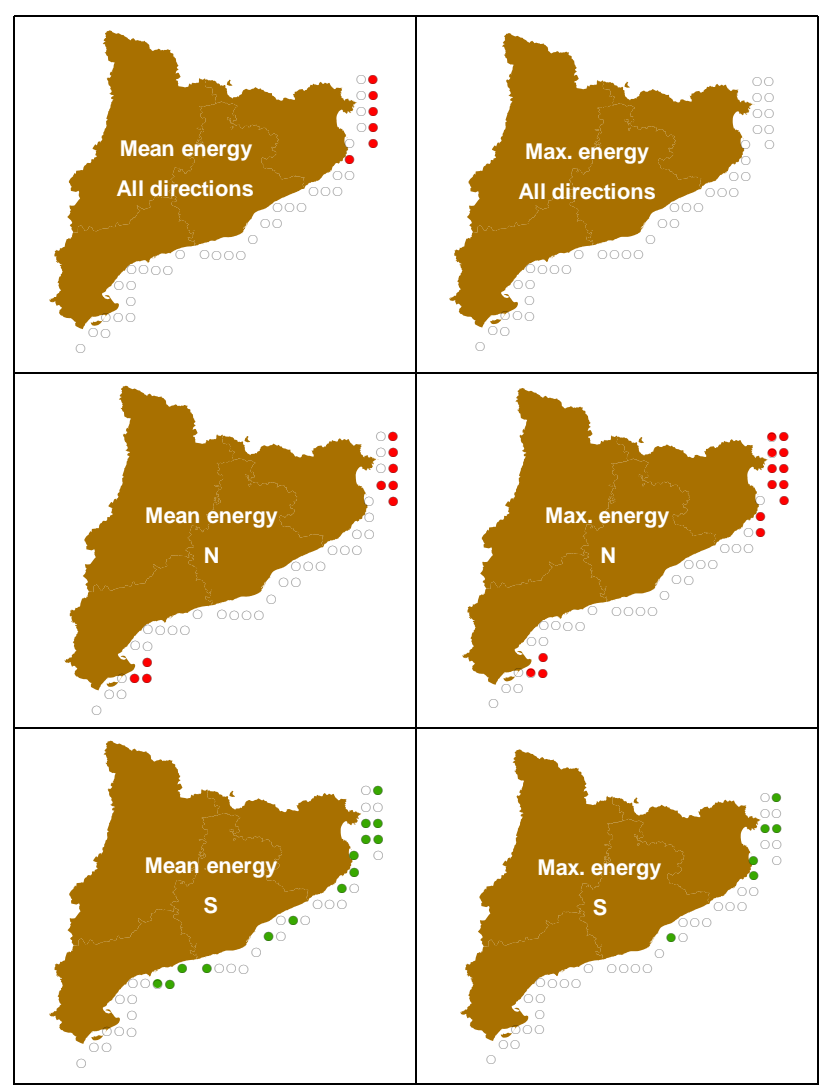

Fig. 2. Trend detection of wave storminess for some directions. Red: negative trend. Blank: no significant trend. Green: positive trend.
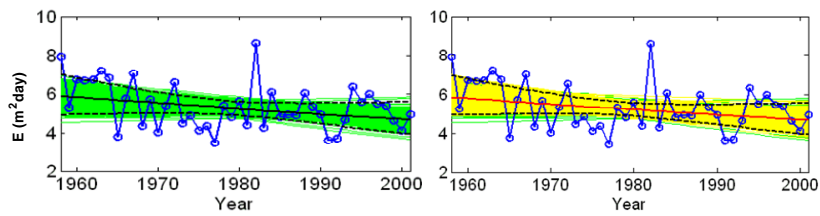

Fig. 3. Examples of evolution of annual mean of storm energy content in all directions (Node 2, see Fig. 1). Blue: time series. Black: linear regression trend. Red: Bayesian mean trend. Green: bootstrapped samples. Yellow: Bayesian posterior samples.

frequent. In terms of wave storminess magnitude, $\mathrm{E}$ is even more important, coming the most energetic storms from $\mathrm{E}$ and being located in the northern coast (although the more frequent direction was $\mathrm{N}$ ).

In order to roughly distinguish sea and swell, the correlation between wind and wave directions has been calculated, using the circular correlation coefficient of Fisher \& Lee (1983). As expected, it is found that northern locations (with a majority of waves coming from $\mathrm{N}$ ) present high values of correlation (0.81-0.92) between both directions, which can be well-explained by the lower $\mathrm{N}$ fetch and, therefore, the larger presence of sea. On the contrary, in the center part, E predominance is related to more mixed sea states, being wave and wind directions poorly correlated $(0.36-0.47)$.

\subsection{Wave storminess}

Figure 2 shows the more significant results of the Mann Kendall test. For the maximum energy there is no general trend but $\mathrm{N}$ wave energy reduction is found in the northern coast whereas $\mathrm{S}$ increase is detected in some nodes along the coast. The temporal tendency of the annual mean energy is similar to the maxima except that there is negative trend considering all directions in the offshore nodes in the most northern coast. It is important to note that the most energetic direction (E) has no significant trend and, therefore, the most severe storms will not tend to change.

Therefore, although in general the temporal trend is null or even negative, in some locations, storm energy content appears to increase for storms coming from S. However, when examining the wave storminess evolution of this type of events, it is found that this rise is more related to annual increase of the number of southern storms rather than their magnitude. In fact, in the first decades of the time series most of the years were without $\mathrm{S}$ storms, which have become a less rare situation in the last years. This increase in the number of storms, as seen in Section 3.3, is translated in a higher percentage of S storms compared to the rest.

To illustrate the comparison between the two methods of trend quantification explained in Section 2, an example (node 2) of energy evolution, for all directions, is given (see Fig. 3). The calculated trend by the two methods is practically the same, since in Fig. 3 the Bayesian trend overlaps the Regression one. In this case, considering the whole period (1958-2001) there is a mean energy reduction of $20 \%$. However, there is large uncertainty due to large energy interannual variability. Note also that the uncertainty obtained by the Bayesian method is similar to the one derived by bootstrapping.

\subsection{Directional frequency}

In terms of annual sector frequency, similarly to wave storminess behaviour, $\mathrm{N}$ tends to decrease and $\mathrm{S}$ to increase but again the most energetic direction remains unchanged. In Fig. 4, the evolution of such frequencies of two nodes, as an example, is illustrated by comparing the annual mean frequency of the past situation (1958-2001) with a future one (2050) obtained by extrapolating the calculated tendency with linear regression. The confidence intervals are meant to indicate the marginal variability but do not integrate the uncertainty between sectors. To catch such interactions, 1000 bootstrapped samples of 2050 are plotted (in yellow, see Fig. 4). In these two illustrated examples, one can highlight the following results (pointed out in Fig. 4 by pink arrows): 

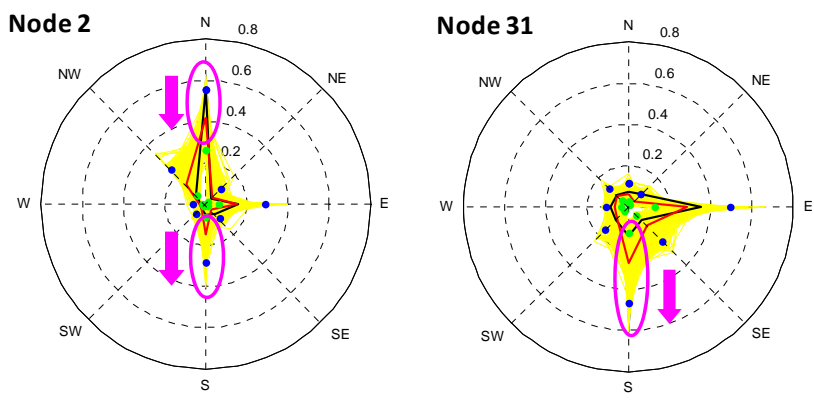

Fig. 4. Trend quantification of annual sector frequency (Nodes 2 and 31, see Fig. 1). Black line: annual mean frequency 1958-2001. Red line: annual mean predicted frequency (2050). Yellow lines: bootstrapped samples (2050). Yellow and Green dots: 95\% confidence intervals (2050).

$\mathrm{N}$ decrease from $56 \%$ to $42 \%$ (node 2 ) and $\mathrm{S}$ increase from $6 \%$ to $15 \%$ (node 2 ) and from $14 \%$ to $27 \%$ (node 31 ).

\section{Conclusions}

Catalan coast is clearly dominated by E wave storms, especially in terms of wave storminess, for which both sea and swell seem to be usually present.

Separating the wave storminess for each direction sector has been found to be relevant since some trends are identified for some directions whereas they are not detected when considering all directions. In general the trends are null or even negative except for some nodes in which the number of southern storms seems to increase. Hence, the mean frequency of events from such direction increases as well with a factor of 2 for some 2050 projections. However, bear in mind that these results are conditional on the used hindcast data which may have some limitations to reproduce the extreme wave climate.

For this type of analysis the regression plus the bootstrap method and the selected Bayesian method present very similar results both in terms of trend magnitude and uncertainty. Although their potential, it is difficult to be accurate in making future predictions with the methods described because the inter-annual variability of the time series is very large.
Acknowledgements. This work has been funded by EU project CIRCE (ref. TST5-CT-2007-036961) and Spanish research project ARCO (ref. no. 200800050084350). The contribution of Puertos del Estado providing datasets is also strongly appreciated. The authors want to thank Juan José Egozcue from UPC for his helpful assistance in assessing the data. The first author is supported by an UPC PhD grant and the Col-legi d'Enginyers de Camins, Canals $i$ Ports - Catalunya (Civil Engineering Association in Catalonia).

Edited by: J. Salat

Reviewed by: one anonymous reviewer

\section{References}

Agresti, A.: Categorical Data Analysis, Second Edition. Wiley, New York, USA, 734 pp., 2002.

Egozcue, J.J., Pawlowsky-Glahn, V., Mateu-Figueras, G., and Barceló-Vidal, C.: Isometric logratio Transformations for Compositional Data Analysis, Math. Geol., 35(3), 279-300, 2003.

Fisher, N. and Lee, A. J.: A correlation coefficient for circular data. Biometrica, 70, 327-332, 1983.

Gomis, D., Ruiz, S., Sotillo, M. G., Álvarez-Fanjul, E., and Terradas, J.: Low frequency Mediterranean sea level variability: The contribution of atmospheric pressure and wind. Global and Planetary Change, 63, 215-229, 2008.

Guedes Soares, C., Carretero Albiach, J. C., Weisse, R., and Alvarez-Fanjul, E.: A 40 years hindcast of wind, sea level and waves in European waters. Proceedings of the 21st International Conference on Offshore Mechanics and Arctic Engineering, Oslo, Norway, 669-675, 2002.

Mendoza, E. T. and Jiménez, J. A.: Storm-Induced Beach Erosion on the Catalonian Coast, J. Coast. Res., Special Issue 48, 81-88, 2006.

Mösso, C., Mestres, M., Sierra, J. P., Sánchez-Arcilla, A., and Goodess, C.: Waves and Surges in the Valencia Gulf. Variability Rather Than Climate Change, J. Coast. Res., Special Issue 56, 243-247, 2009.

Musić, S. and Nicković, S.: 44-year wave hindcast for the Eastern Mediterranean, Coast. Eng., 55, 872-880, 2008.

Ratsimandresy, A. W., Sotillo, M. G., Carretero Albiach, J. C., Álvarez Fanjul, E., and Haiji, H.: A 44-year high resolution ocean and atmospheric hindcast for the Mediterranean Basin developed within the HIPOCAS project, Coast. Eng., 55, 827-842, 2008 . 